\title{
Electromagnetic Induction in an Irregular Layer Overlying the Earth. The 2nd paper: A Semi-infinite Medium of Finite Conductivity Having an Undulatory Surface
}

\author{
Naoto OHSHIMAN and Tsuneji RIKITAKE \\ Department of Earth Sciences, College of Humanities and Sciences, Nihon \\ University, Sakurajosui, Setagaya-ku, Tokyo, Japan
}

(Received December 1, 1984; Revised January 21, 1985)

Electromagnetic induction by a uniform magnetic field in a semi-infinite medium of finite conductivity having a sinusoidal undulation at the surface is studied. The source field is assumed to be parallel to the mean surface. The apparent resistivity $\left(\rho_{\mathrm{a}}\right)$ vs. period of variation $(T)$ relation is obtained at various points over the undulating surface by means of conventional magneto-telluric technique. The phase vs. $T$ and the transfer function vs. $T$ relations are also obtained from the electric and magnetic fields studied in this paper.

It turns out that the $\rho_{\mathrm{a}}-T$ relation is little affected by the surface undulation. The order of magnitude of $\rho_{\mathrm{a}}$ value at various points over the surface does not vary although $\rho_{\mathrm{a}}$ for a longer period variation takes on a relatively lower value at a depressed portion of the undulatory surface. On the other hand, the transfer function vs. $T$ relation differs from observation point to observation point depending upon the position of observation point on the undulatory topography.

\section{Introduction}

RIKITAKE and OHSHIMAN (1985) studied electromagnetic induction by a uniform inducing field, either parallel or perpendicular to the earth's surface, in an earth model consisting of a superficial thin layer over a non-conductor underlain by an undulating perfect conductor using a method developed by RIKITAKE $(1965,1968)$. Although the model is a somewhat artificial one, it is shown that, if we interpret the $\rho_{\mathrm{a}}-T$ relation obtained basing on a two-layer model to which the conventional magneto-tellurics (MT) is applied, an underground structure, which is significantly different from that of the original model, is obtained.

In view of the above conclusion obtained in the first paper concerning electromagnetic induction in an irregular layer overlying the earth, the authors further intend to study electromagnetic induction in a more realistic model which approximates the actual earth. In this paper, first of all, electromagnetic induction in a semi-infinite medium of finite conductivity having an undulatory sur- 
face will be studied in the hope of making the topographical effect on an MT result, for the case of mountain range, valley-like depression and the like, clear.

It is highly important to estimate the effect of topography on results of MT observation because investigations into the electric conductivity distribution in the earth's crust have recently been carried out very intensively in the various topographical' regions in Japan utilizing the MT technique (e.g., YUKUTAKE et al., 1983; Research Group for Crustal Resistivity Structure, Japan, 1983).

It is clear that the MT method can lead us to an exact solution in the case of an earth structure which is laterally uniform provided the inducing field is fairly uniform (e.g., RIKITAKE, 1966). Such a condition, however, is hardly fulfilled in an actual case, especially in the Japan Islands where the topography is so complicated that most measurements have to be made in a mountainous area or near the sea coast. Therefore, the authors would like to emphasize the importance of the evaluation of the effect of topography on MT observation results.

The authors hope that the study of electromagnetic induction, that will be developed in this paper, may be helpful for interpreting actual results of MT measurement which is carried out over an earth having a surface of irregular shape.

The theory developed here is similar to that in RIKITAKE $(1965,1968)$ and RIKITAKE and OHSHIMAN (1985). Magnetic potential of the induced field is expressed in a form of Fourier series, and the coefficients are determined by the boundary conditions in units of the amplitude of the prescribed inducing field. The dependency of apparent resistivity and transfer function on period of variation will then be obtained with the aid of these coefficients.

\section{Theory}

Let us assume that a time-dependent uniform magnetic field is applied to a semi-infinite conductor having an undulatory surface which is defined by $z=f(x)$. The coordinate axes are taken as seen in Fig. 1. Function $f(x)$ is defined for

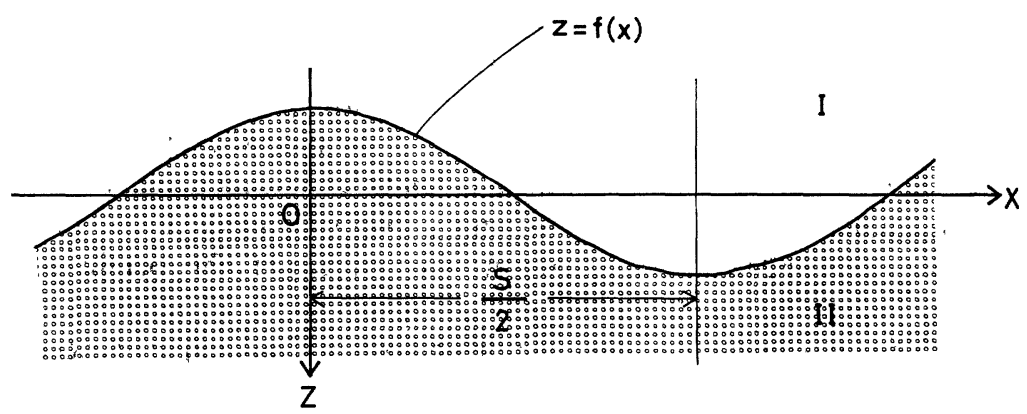

Fig. 1. The coordinates and system of conductor. Region I is insulating, while Region II is a semiinfinite medium of finite conductivity. The undulatory boundáry between Regions I and II is given by $z=f(x)$. 
$-s / 2 \leqq x \leqq s / 2$. Outside this domain, the same surface shape is assumed to repeat periodically with respect to $x$.

We can define a magnetic potential $W$ in the free space above the conductor. $W$ satisfies

$$
\nabla^{2} W=0
$$

of which the typical solution fitting in the present problem is given by

$$
\left.W=-H_{0} x+\sum_{n=0}^{\infty} A_{n} \mathrm{e}^{(2 \pi / l) n z} \sin \frac{2 \pi}{l} n x \quad \text { (for Region } \mathrm{I}\right)
$$

in which $H_{0}$ is the uniform magnetic field applied from outside and the term expressed by the series denotes the magnetic potential arising from the electric currents induced in the conductor. $A_{0}$ is assumed to be zero without losing generality. When $f(x)$ is an even function, it is obvious that the magnetic potential is to be an odd function of $x$ and so only terms proportional to $\sin (2 \pi / l) n x$ are involved in (2). $l$ denotes the longest wavelength involved in the problem, so that we assume $s \leqq l$.

In the conductor of which the electrical conductivity is $\sigma$, we can define a vector potential which has only the $y$ component in the present problem. Denoting that component by $A_{y}$, the differential equation satisfied by $A_{y}$ is written as

$$
4 \pi \sigma \frac{\partial A_{y}}{\partial t}=\nabla^{2} A_{y}
$$

in which $t$ is time. Time operator $\partial / \partial t$ will be written as $p$ hereafter.

In that case the typical solution, which does not become infinite at $z \rightarrow \infty$, can be given by

$$
A_{y}=\sum_{n=0}^{\infty} B_{n} \mathrm{e}^{-\sqrt{((2 \pi / l) n)^{2}+\gamma^{2}} z} \cos \frac{2 \pi}{l} n x \quad \text { (for Region II) }
$$

where

$$
\gamma^{2}=4 \pi \sigma p
$$

Meanwhile, the magnetic permeability is assumed as unity in the electromagnetic unit.

The magnetic field components in the free space and the conductor are then obtained respectively as 


$$
\left.\begin{array}{l}
H_{x}=H_{0}-\frac{2 \pi}{l} \sum_{n} n A_{n} \mathrm{e}^{(2 \pi / l) n z} \cos \frac{2 \pi}{l} n x \\
H_{z}=-\frac{2 \pi}{l} \sum_{n} n A_{n} \mathrm{e}^{(2 \pi / l) n z} \sin \frac{2 \pi}{l} n x
\end{array}\right\} . \quad \text { (for Region I) }
$$

The boundary condition at $z=f(x)$ requires that the tangential component of magnetic field is continuous there. The continuity of the normal component of magnetic flux density should also hold good. As the magnetic permeability is assumed as unity throughout the space, the normal component of magnetic field becomes continuous. It is then obvious that both the $x$ and $z$ components of magnetic field are to be continuous at the boundary.

We thus have

$$
\begin{aligned}
H_{0}-\frac{2 \pi}{l} \sum_{n} n A_{n} \mathrm{e}^{(2 \pi / l) n f(x)} \cos \frac{2 \pi}{l} n x= & \sum_{n} \sqrt{\left(\frac{2 \pi}{l} n\right)^{2}+\gamma^{2} B_{n} \mathrm{e}^{-\sqrt{((2 \pi / l) n)^{2}+\gamma^{2}} f(x)}} \\
& \cos \frac{2 \pi}{l} n x \\
\sum_{n} n A_{n} \mathrm{e}^{(2 \pi / l) n f(x)} \sin \frac{2 \pi}{l} n x= & \sum_{n} n B_{n} \mathrm{e}^{-\sqrt{((2 \pi / l) n)^{2}+\gamma^{2}} f(x)} \sin \frac{2 \pi}{l} n x .
\end{aligned}
$$

When both sides of the two equations of (8) are multiplied respectively by $\cos (2 \pi / l) N x$ and $\sin (2 \pi / l) N x$ and integrated from $-l / 2$ to $l / 2$ with respect to $x$, we obtain

$$
\begin{aligned}
& \sum_{n}\left(\frac{2 \pi}{l} n A_{n} P_{n N}+\sqrt{\left(\frac{2 \pi}{l} n\right)^{2}+\gamma^{2}} B_{n} T_{n N}\right)=l \in H_{0}, \\
& \sum_{n} n\left(A_{n} Q_{n N}-B_{n} U_{n N}\right)=0
\end{aligned}
$$

where

$$
\begin{gathered}
\epsilon=1 \text { for } N=0, \quad \in=0 \text { for } N \neq 0 \\
P_{n N}=\int_{-l / 2}^{l / 2} \mathrm{e}^{(2 \pi / l) n f(x)} \cos \frac{2 \pi}{l} n x \cos \frac{2 \pi}{l} N x \mathrm{~d} x
\end{gathered}
$$




$$
\left.\begin{array}{rl}
Q_{n N} & =\int_{-l / 2}^{l / 2} \mathrm{e}^{(2 \pi / l) n f(x)} \sin \frac{2 \pi}{l} n x \sin \frac{2 \pi}{l} N x \mathrm{~d} x \\
T_{n N} & =\int_{-l / 2}^{l / 2} \mathrm{e}^{-\sqrt{((2 \pi / l) n)^{2}+\gamma^{2} f(x)}} \cos \frac{2 \pi}{l} n x \cos \frac{2 \pi}{l} N x \mathrm{~d} x \\
U_{n N} & =\int_{-l / 2}^{l / 2} \mathrm{e}^{-\sqrt{((2 \pi / l) n)^{2}+\gamma^{2}} f(x)} \sin \frac{2 \pi}{l} n x \sin \frac{2 \pi}{l} N x \mathrm{~d} x
\end{array}\right\} .
$$

When we truncate the series involved in (9) and (10) at a certain level of $n$, we have a set of simultaneous equations solving which we can obtain $A_{n}$ 's and $B_{n}$ 's. With these coefficients, we can determine the magnetic and electric fields associated with the present electromagnetic induction process.

3. Magnetic and Electric Fields over the Surface

The process discussed in the last section is an electromagnetic induction by an external magnetic field of which the $x$ and $z$ components are given by

$$
\left.\begin{array}{l}
H_{e x}=H_{0} \\
H_{e z}=0
\end{array}\right\} \text {. }
$$

Meanwhile, the components of induced magnetic field at the surface are given by

$$
\left.\begin{array}{l}
H_{i x}=-\frac{2 \pi}{l} \sum_{n} A_{n} \mathrm{e}^{(2 \pi / l) n f(x)} \cos \frac{2 \pi}{l} n x \\
H_{i z}=-\frac{2 \pi}{l} \sum_{n} n A_{n} \mathrm{e}^{(2 \pi / l) n f(x)} \sin \frac{2 \pi}{l} n x
\end{array}\right\}
$$

in which $A_{n}$ 's are to be obtained by the theory developed in the last section. Equations (12) and (13) make the relationship between the inducing and induced fields clear. It is obviously seen that a uniform inducing field excites a nonuniform induced field.

The transfer function, that is defined by the ratio of the vertical magnetic field to the horizontal one, is given by

$$
\frac{H_{z}}{H_{x}}=\frac{-\frac{2 \pi}{l} \sum_{n} n A_{n} \mathrm{e}^{(2 \pi / l) n f(x)} \sin \frac{2 \pi}{l} n x}{H_{0}-\frac{2 \pi}{l} \sum_{n} n A_{n} \mathrm{e}^{(2 \pi / l) n f(x)} \cos \frac{2 \pi}{l} n x} .
$$


The $y$ component of electric field is given by

$$
E_{y}=-\frac{\partial A_{y}}{\partial t}
$$

so that we have

$$
E_{y}=-p \sum_{n} B_{n} \mathrm{e}^{-\sqrt{((2 \pi / l) n)^{2}+\gamma^{2}} f(x)} \cos \frac{2 \pi}{l} n x
$$

at the surface.

The ratio of the $y$ component of electric field to the $x$ component of magnetic field becomes

$$
\frac{E_{y}}{H_{x}}=-\frac{p \sum_{n} B_{n} \mathrm{e}^{-\sqrt{((2 \pi / l) n)^{2}+\gamma^{2} f(x)}} \cos \frac{2 \pi}{l} n x}{H_{0}-\frac{2 \pi}{l} \sum_{n} n A_{n} \mathrm{e}^{(2 \pi / l) n f(x)} \cos \frac{2 \pi}{l} n x}
$$

which is useful for estimating the apparent resistivity of the conductor by the magneto-telluric method.

4. Purely Periodic Time Variation

Let us assume that the time variation is purely periodic with an angular frequency $\omega$. In that case we put

$$
p=i \omega \quad(i=\sqrt{-1}) .
$$

In general $A_{n}$ 's and $B_{n}$ 's are complex, so that let us put

$$
\left.\begin{array}{l}
A_{n}=\bar{A}_{n}+i A_{n}^{*} \\
B_{n}=\bar{B}_{n}+i B_{n}^{*}
\end{array}\right\}
$$

where $\bar{A}_{n}, A_{n}^{*}, \bar{B}_{n}$ and $B_{n}^{*}$ are all real quantities.

From (5), we obtain

$$
\gamma^{2}=i \Omega^{2} / l^{2}
$$

where

$$
\Omega^{2}=4 \pi \sigma \omega l^{2}
$$


in which $\Omega$ is customarily called the induction parameter.

Making use of (20), we obtain

$$
\sqrt{\left(\frac{2 \pi}{l} n\right)^{2}+\gamma^{2}}=\left(a^{2}+b^{2}\right)^{1 / 4}\left(\cos \frac{\theta}{2}+i \sin \frac{\theta}{2}\right)
$$

where

$$
\left.\begin{array}{rl}
a & =\left(\frac{2 \pi n}{l}\right)^{2} \\
b & =\left(\frac{\Omega}{l}\right)^{2} \\
\tan \theta & =\frac{b}{a}
\end{array}\right\} .
$$

It then follows that

$$
\left.\begin{array}{rl}
T_{n N} & =\bar{T}_{n N}+i T_{n N}^{*} \\
U_{n N} & =\bar{U}_{n N}+i U_{n N}^{*}
\end{array}\right\}
$$

where

$$
\begin{aligned}
\bar{T}_{n N}= & \int_{-l / 2}^{l / 2} \mathrm{e}^{-\left(a^{2}+b^{2}\right)^{1 / 4} \cos (\theta / 2) f(x)} \cos \left[\left(a^{2}+b^{2}\right)^{1 / 4} \sin \frac{\theta}{2} f(x)\right] \\
& \cos \frac{2 \pi}{l} n x \cos \frac{2 \pi}{l} N x \mathrm{~d} x \\
T_{n N}^{*}= & -\int_{-l / 2}^{l / 2} \mathrm{e}^{-\left(a^{2}+b^{2}\right)^{1 / 4} \cos (\theta / 2) f(x)} \sin \left[\left(a^{2}+b^{2}\right)^{1 / 4} \sin \frac{\theta}{2} f(x)\right] \\
& \cos \frac{2 \pi}{l} n x \cos \frac{2 \pi}{l} N x \mathrm{~d} x \\
\bar{U}_{n N}= & \int_{-l / 2}^{l / 2} \mathrm{e}^{-\left(a^{2}+b^{2}\right)^{1 / 4} \cos (\theta / 2) f(x)} \cos \left[\left(a^{2}+b^{2}\right)^{1 / 4} \sin \frac{\theta}{2} f(x)\right] \\
& \sin \frac{2 \pi}{l} n x \sin \frac{2 \pi}{l} N x \mathrm{~d} x \\
U_{n N}^{*}= & -\int_{-l / 2}^{l / 2} \mathrm{e}^{-\left(a^{2}+b^{2}\right)^{1 / 4} \cos (\theta / 2) f(x)} \sin \left[\left(a^{2}+b^{2}\right)^{1 / 4} \sin \frac{\theta}{2} f(x)\right] \\
& \sin \frac{2 \pi}{l} n x \sin \frac{2 \pi}{l} N x \mathrm{~d} x
\end{aligned}
$$


With the aid of (22) and (25), Equations (9) and (10) can be rewritten as

$$
\begin{gathered}
\sum_{n}\left[\frac{2 \pi}{l} n\left(\bar{A}_{n}+i A_{n}^{*}\right) P_{n N}+\left(a^{2}+b^{2}\right)^{1 / 4}\left(\cos \frac{\theta}{2}+i \sin \frac{\theta}{2}\right)\right. \\
\left.\left(\bar{B}_{n}+i B_{n}^{*}\right)\left(\bar{T}_{n N}+i T_{n N}^{*}\right)\right]=l \in H_{0} \\
\sum_{n}\left[\left(\bar{A}_{n}+i A_{n}^{*}\right) Q_{n N}-\left(\bar{B}_{n}+i B_{n}^{*}\right)\left(\bar{U}_{n N}+i U_{n N}^{*}\right)\right]=0
\end{gathered}
$$

Equating the real and imaginary parts on both sides of the equations involved in (26), we obtain

$$
\left.\begin{array}{l}
\sum_{n}\left[\frac{2 \pi}{l} n \bar{A}_{n} P_{n N}+C_{n N} \cos \frac{\theta}{2}-D_{n N} \sin \frac{\theta}{2}\right]=l \in H_{0} \\
\sum_{n}\left[\frac{2 \pi}{l} n A_{n}^{*} P_{n N}+C_{n N} \sin \frac{\theta}{2}+D_{n N} \cos \frac{\theta}{2}\right]=0
\end{array}\right\}
$$

where

$$
\left.\begin{array}{l}
C_{n N}=\left(a^{2}+b^{2}\right)^{1 / 4}\left(\bar{B}_{n} \bar{T}_{n N}-B_{n}^{*} T_{n N}^{*}\right) \\
D_{n N}=\left(a^{2}+b^{2}\right)^{1 / 4}\left(\bar{B}_{n} T_{n N}^{*}+B_{n}^{*} \bar{T}_{n N}\right)
\end{array}\right\}
$$

and

$$
\left.\begin{array}{l}
\sum_{n} n\left[\bar{A}_{n} Q_{n N}-\bar{B}_{n} \bar{U}_{n N}+B_{n}^{*} U_{n N}^{*}\right]=0 \\
\sum_{n} n\left[A_{n}^{*} Q_{n N}-\bar{B}_{n} U_{n N}^{*}-B_{n}^{*} \bar{U}_{n N}\right]=0
\end{array}\right\} .
$$

(27) and (29) provide a set of simultaneous equations solving which we can obtain $\bar{A}_{n}$ 's, $A_{n}^{*}$ 's, $\bar{B}_{n}$ 's and $B_{n}^{*}$ 's on the condition that $n$ is truncated at a suitable level.

5. Semi-Infinite Conductor with a Surface of Sinusoidal Undulation

Let us assume that

$$
f(x)=-h \cos \frac{2 \pi}{s} x
$$


which indicates that the surface of the conducting medium undulates in a sinusoidal way with a wavelength $s$.

Putting (30) into the first two equations of (11) and (25), we obtain

$$
\begin{aligned}
P_{n N}= & \int_{-l / 2}^{l / 2} \mathrm{e}^{-(2 \pi / l) n h \cos (2 \pi / s) x} \cos \frac{2 \pi}{l} n x \cos \frac{2 \pi}{l} N x \mathrm{~d} x \\
Q_{n N}= & \int_{-l / 2}^{l / 2} \mathrm{e}^{-(2 \pi / l) n h \cos (2 \pi / s) x} \sin \frac{2 \pi}{l} n x \sin \frac{2 \pi}{l} N x \mathrm{~d} x \\
\bar{T}_{n N}= & \int_{-l / 2}^{l / 2} \mathrm{e}^{h\left(a^{2}+b^{2}\right)^{1 / 4} \cos (\theta / 2) \cos (2 \pi / s) x} \cos \left[h\left(a^{2}+b^{2}\right)^{1 / 4} \sin \frac{\theta}{2} \cos \frac{2 \pi}{s} x\right] \\
& \cos \frac{2 \pi}{l} n x \cos \frac{2 \pi}{l} N x \mathrm{~d} x \\
T_{n N}^{*}= & \int_{-l / 2}^{l / 2} \mathrm{e}^{h\left(a^{2}+b^{2}\right)^{1 / 4} \cos (\theta / 2) \cos (2 \pi / s) x} \sin \left[h\left(a^{2}+b^{2}\right)^{1 / 4} \sin \frac{\theta}{2} \cos \frac{2 \pi}{s} x\right] \\
& \cos \frac{2 \pi}{l} n x \cos \frac{2 \pi}{l} N x \mathrm{~d} x \\
\bar{U}_{n N}= & \int_{-l / 2}^{l / 2} \mathrm{e}^{h\left(a^{2}+b^{2}\right)^{1 / 4} \cos (\theta / 2) \cos (2 \pi / s) x} \cos \left[h\left(a^{2}+b^{2}\right)^{1 / 4} \sin \frac{\theta}{2} \cos \frac{2 \pi}{s} x\right] \\
& \sin \frac{2 \theta}{l} n x \sin \frac{2 \pi}{l} N x \mathrm{~d} x \\
U_{n N}^{*}= & \int_{-l / 2}^{l / 2} \mathrm{e}^{h\left(a^{2}+b^{2}\right)^{1 / 4} \cos (\theta / 2) \cos (2 \pi / s) x} \sin \left[h\left(a^{2}+b^{2}\right)^{1 / 4} \sin \frac{\theta}{2} \cos \frac{2 \pi}{s} x\right] \\
& \sin \frac{2 \pi}{l} n x \sin \frac{2 \pi}{l} N x \mathrm{~d} x
\end{aligned}
$$

If we specify $s, l$, and $h$, the integrals involved in (31) can be calculated for various combinations of $n$ and $N$. Parameters $l, s, \sigma$ and $\Omega$ should be properly taken according to the electrical structure considered.

Let us study two induction models of which the parameters are shown in Table 1. Solving simultaneous Eqs. (27) and (29) for $n=0,1,-12, \bar{A}_{n}, A_{n}^{*}$, $\bar{B}_{n}$ and $B_{n}^{*}$ are obtained in units of $H_{0}$ as shown in Tables 2 and 3 for $T=0.143$

Table 1. Parameters of the induction models shown in Fig. 1.

\begin{tabular}{lcc}
\hline Parameter & Model I & Model II \\
\hline$s(=l)(\mathrm{km})$ & 10.0 & 10.0 \\
$h(\mathrm{~m})$ & 100.0 & 1000.0 \\
$\sigma($ e.m.u. $)$ & $10^{-13}$ & $10^{-13}$ \\
$1 / \sigma(\Omega$-m $)$ & 100 & 100 \\
\hline
\end{tabular}


Table 2. $\bar{A}_{n}, A_{n}^{*}, \bar{B}_{n}$ and $B_{n}^{*}$ of Model $\mathrm{I}$ in units of $H_{0}$ for the two periods. (a) $T=0.143 \mathrm{~s}$

\begin{tabular}{|c|c|c|c|c|}
\hline$n$ & $\bar{A}_{n}$ & $A_{n}^{*}$ & $\bar{B}_{n}$ & $B_{n}^{*}$ \\
\hline 0 & 0.0 & 0.0 & $0.95034 \mathrm{D}+05$ & $-0.95056 \mathrm{D}+05$ \\
\hline 1 & $-0.45840 \mathrm{D}+04$ & $-0.25945 \mathrm{D}+04$ & $-0.47586 \mathrm{D}+04$ & $-0.25902 \mathrm{D}+04$ \\
\hline 2 & $-0.89757 \mathrm{D}+02$ & $-0.74462 \mathrm{D}+02$ & $0.41832 \mathrm{D}+02$ & $0.57043 \mathrm{D}+02$ \\
\hline 3 & $-0.29498 \mathrm{D}+01$ & $-0.29371 D+01$ & $-0.11525 \mathrm{D}+10$ & $-0.14886 \mathrm{D}+01$ \\
\hline 4 & $-0.12401 \mathrm{D}+00$ & $-0.13846 \mathrm{D}+00$ & $0.48538 \mathrm{D}-01$ & $0.54497 \mathrm{D}-01$ \\
\hline 5 & $-0.64979 \mathrm{D}-02$ & $-0.72975 \mathrm{D}-02$ & $-0.30981 \mathrm{D}-02$ & $-0.24266 \mathrm{D}-02$ \\
\hline 6 & $-0.19316 \mathrm{D}-04$ & $-0.41902 \mathrm{D}-03$ & $0.78854 \mathrm{D}-03$ & $0.12132 \mathrm{D}-03$ \\
\hline 7 & $-0.26888 \mathrm{D}-03$ & $-0.23319 D-04$ & $-0.60964 \mathrm{D}-03$ & $-0.63194 \mathrm{D}-05$ \\
\hline 8 & $0.20411 \mathrm{D}-03$ & $-0.26814 \mathrm{D}-05$ & $0.56145 \mathrm{D}-03$ & $0.46195 \mathrm{D}-06$ \\
\hline 9 & $-0.17135 \mathrm{D}-03$ & $0.62779 \mathrm{D}-06$ & $-0.53085 \mathrm{D}-03$ & $-0.35311 \mathrm{D}-06$ \\
\hline 10 & $0.14597 \mathrm{D}-03$ & $-0.50660 \mathrm{D}-06$ & $0.50601 \mathrm{D}-03$ & $0.43349 \mathrm{D}-06$ \\
\hline 11 & $-0.11313 \mathrm{D}-03$ & $0.35131 \mathrm{D}-06$ & $-0.46398 \mathrm{D}-03$ & $-0.29030 \mathrm{D}-06$ \\
\hline \multirow[t]{2}{*}{12} & $0.15820 \mathrm{D}-03$ & $-0.31070 \mathrm{D}-06$ & $0.31565 \mathrm{D}-03$ & $-0.12304 \mathrm{D}-06$ \\
\hline & (b) $T=0.0333 \mathrm{~s}$ & & & \\
\hline$n$ & $\bar{A}_{n}$ & $A_{n}^{*}$ & $\bar{B}_{n}$ & $B_{n}^{*}$ \\
\hline 0 & 0.0 & 0.0 & $0.45948 D+05$ & $-0.45715 \mathrm{D}+05$ \\
\hline 1 & $-0.71522 \mathrm{D}+04$ & $-0.20570 \mathrm{D}+04$ & $-0.71339 D+04$ & $-0.20613 D+04$ \\
\hline 2 & $-0.18114 \mathrm{D}+03$ & $-0.81381 \mathrm{D}+02$ & $0.90971 \mathrm{D}+02$ & $0.19070 \mathrm{D}+03$ \\
\hline 3 & $-0.68673 \mathrm{D}+01$ & $-0.40112 \mathrm{D}+01$ & $-0.75963 \mathrm{D}+00$ & $-0.54239 \mathrm{D}+01$ \\
\hline 4 & $-0.31382 \mathrm{D}+00$ & $-0.21896 \mathrm{D}+00$ & $0.24400 \mathrm{D}-01$ & $0.18188 \mathrm{D}+00$ \\
\hline 5 & $-0.16536 \mathrm{D}-01$ & $-0.12730 \mathrm{D}-01$ & $-0.22701 \mathrm{D}-02$ & $-0.75692 \mathrm{D}-02$ \\
\hline 6 & $-0.58781 \mathrm{D}-03$ & $-0.79227 \mathrm{D}-03$ & $0.76388 \mathrm{D}-03$ & $0.35912 \mathrm{D}-03$ \\
\hline 7 & $-0.30224 \mathrm{D}-03$ & $-0.42012 \mathrm{D}-04$ & $-0.60896 \mathrm{D}-03$ & $-0.17511 \mathrm{D}-04$ \\
\hline 8 & $0.20182 \mathrm{D}-03$ & $-0.79629 D-05$ & $0.56136 \mathrm{D}-03$ & $0.13890 \mathrm{D}-05$ \\
\hline 9 & $-0.17138 \mathrm{D}-03$ & $0.29079 \mathrm{D}-05$ & $-0.53080 \mathrm{D}-03$ & $-0.14767 \mathrm{D}-05$ \\
\hline 10 & $0.14590 \mathrm{D}-03$ & $-0.21545 \mathrm{D}-05$ & $0.50599 \mathrm{D}-03$ & $0.18558 \mathrm{D}-05$ \\
\hline 11 & $-0.11310 \mathrm{D}-03$ & $0.15056 \mathrm{D}-05$ & $-0.46397 \mathrm{D}-03$ & $-0.12441 \mathrm{D}-05$ \\
\hline 12 & $0.15817 \mathrm{D}-03$ & $-0.13309 \mathrm{D}-05$ & $0.31564 \mathrm{D}-03$ & $-0.56561 \mathrm{D}-06$ \\
\hline
\end{tabular}

and $0.0333 \mathrm{~s}$ as examples. Coefficients for other periods can readly be obtained in a similar fashion although they are not given here.

With the aid of $\bar{A}_{n}, A_{n}^{*}, \bar{B}_{n}$ and $B_{n}^{*}$ thus determined, the apparent resistivities at a number of points on the earth's surface are calculated from (17) and the well-known formula in MT, i.e., $\rho_{\mathrm{a}}=0.2 T\left|E_{y} / H_{x}\right|^{2}$, as shown in Fig. 2. $\rho_{\mathrm{a}}, T$, $E_{y}$ and $H_{x}$ are measured in units of ohm meter, second, $\mathrm{mV} / \mathrm{km}$ and $\mathrm{nT}$, respectively. Looking at these curves, we see that $\rho_{\mathrm{a}}$ calculated for Model II deviates very little from the resistivity assumed for the semi-infinite earth. This means that an MT measurement at any portion of the undulatory surface leads to an approximately correct value of resistivity, $\rho_{\mathrm{a}}$ being different from the true resistivity only by factor one. It is also seen in the figure that $\rho_{\mathrm{a}}$ takes on an almost constant value regardless of $T$ except for extremely short periods. In the case of 
Table 3. $\bar{A}_{n}, A_{n}^{*}, \bar{B}_{n}$ and $B_{n}^{*}$ of Model II in units of $H_{0}$ for the two periods.

(a) $T=0.143 \mathrm{~s}$

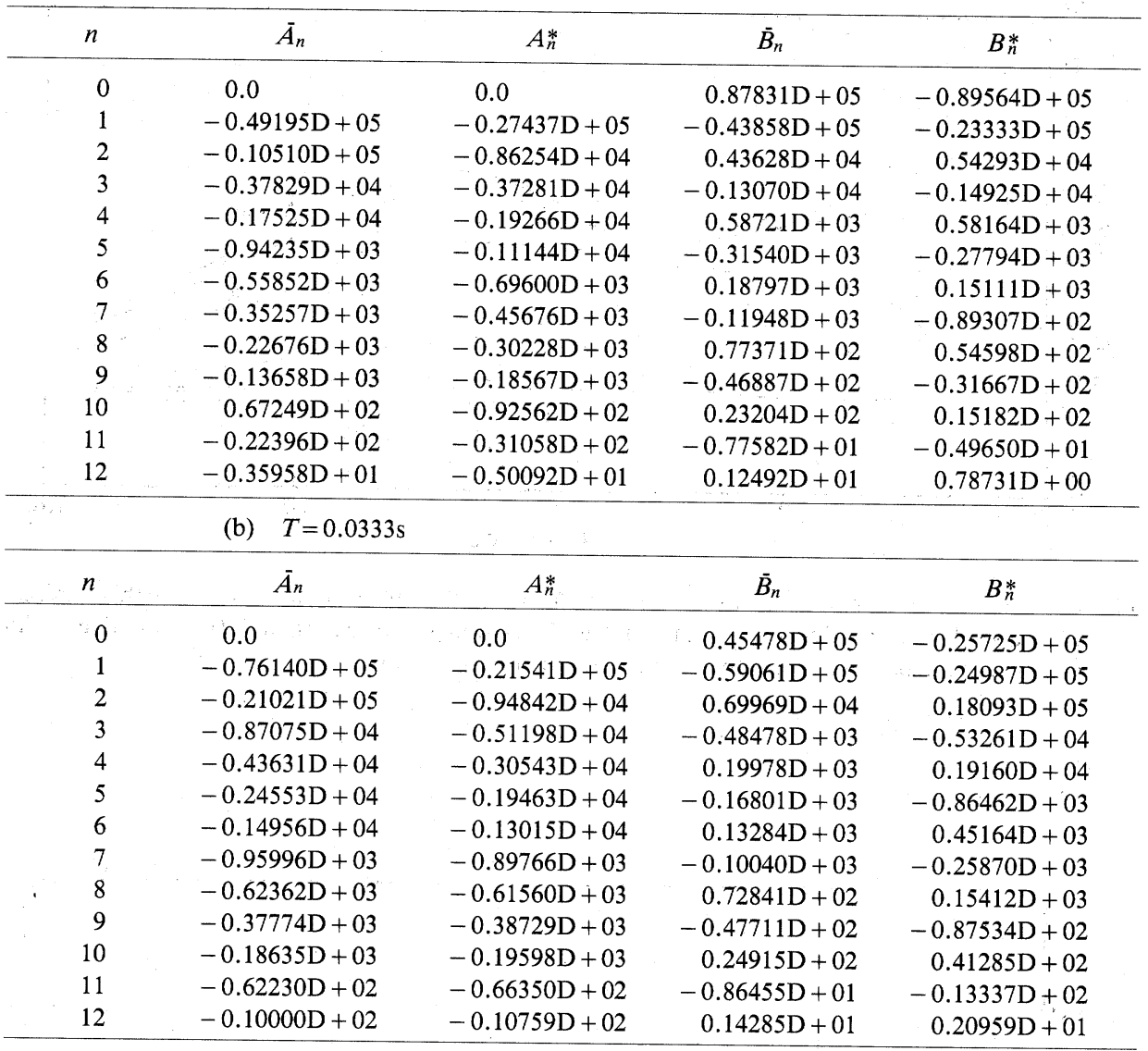

Model I, for which the undulation amplitude is only one-tenth of that for Model II, $\rho_{\mathrm{a}}$ at any point is constant for any period, so that no effect of surface topography can be seen in this case.

Figure 3 shows the phase- $T$ relation between $E_{y}$ and $H_{x}$ at a number of points on the earth's surface. We notice some effect of the undulatory surface for shorter-period variations though not so remarkable. The theory of electromagnetic induction by a uniform magnetic field in a semi-infinite conductor having a flat surface postulates that the component of the inducing magnetic field perpendicular to the surface is always cancelled out by that of the induced field and the components of the induced magnetic field parallel to the surface are indeterminate (e.g., RIKITAKE, 1966). It is shown, however, that the relations between magnetic and electric fields in the earth are determinate. We see 


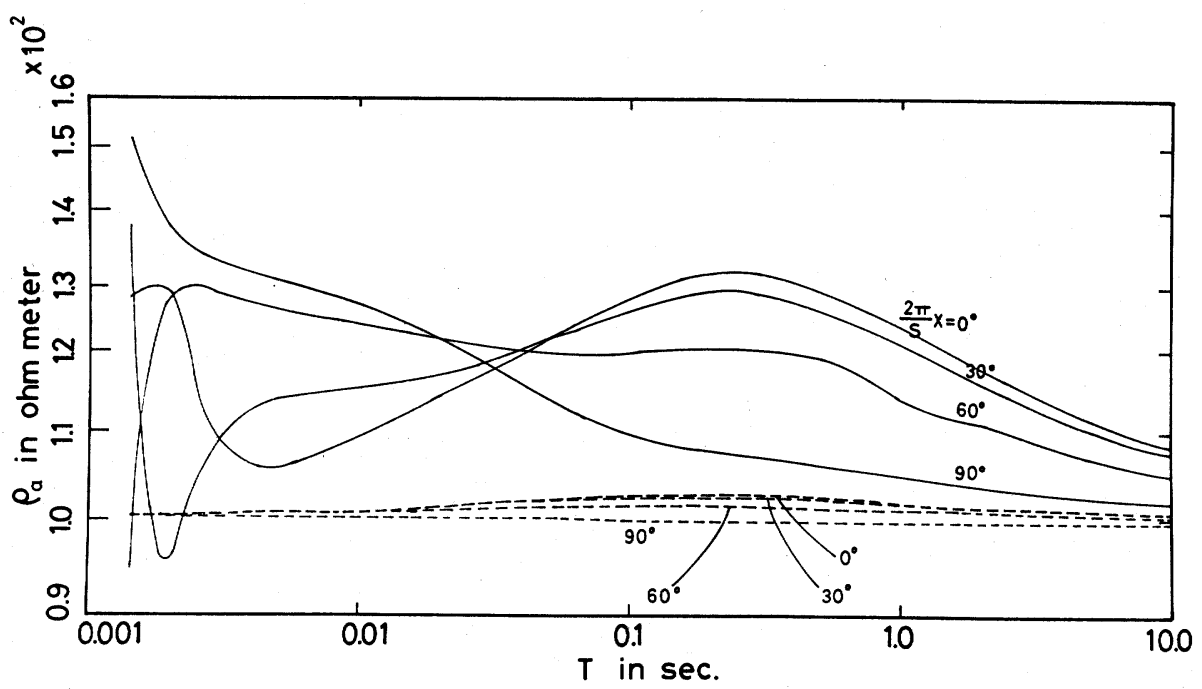

Fig. 2. The $\rho_{\mathrm{a}}-T$ curves at four points, i.e. $(2 \pi / s) x=0^{\circ}, 30^{\circ}, 60^{\circ}$ and $90^{\circ}$, on the earth's surface. The dashed and solid curves indicate the $\rho_{\mathrm{a}}-T$ relation of Models I and II, respectively. An inducing field parallel to the earth's mean surface is assumed.

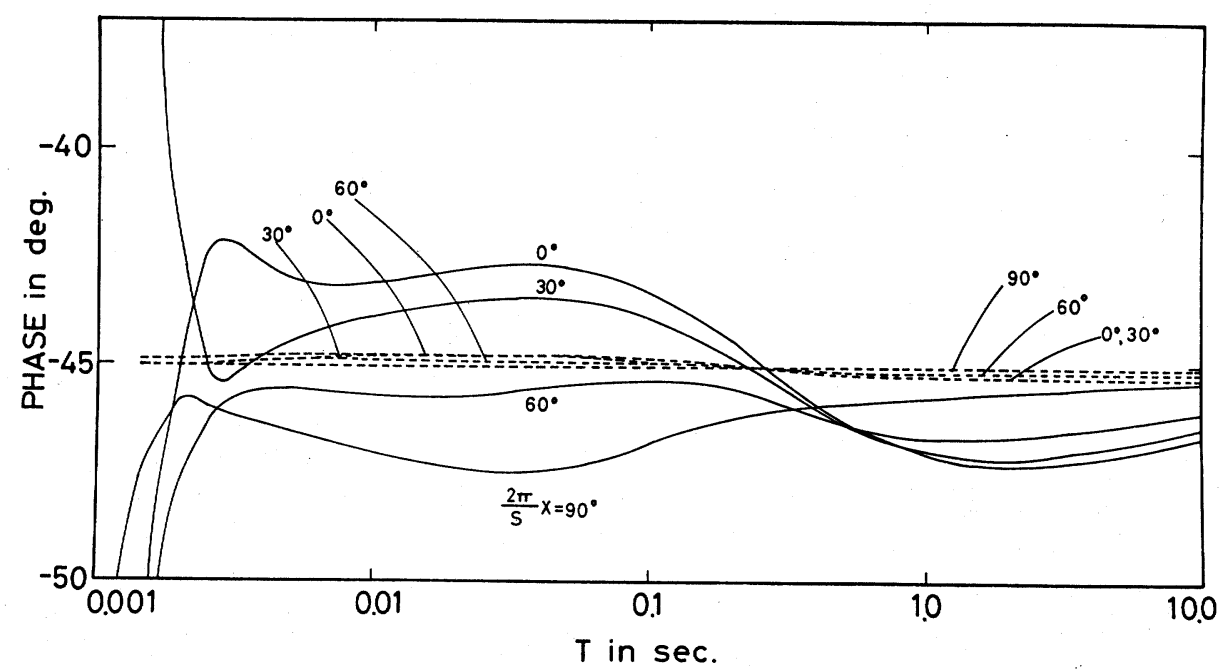

Fig. 3. The phase difference vs. $T$ curves at four points, i.e. $(2 \pi / s) x=0^{\circ}, 30^{\circ}, 60^{\circ}$ and $90^{\circ}$, on the earth's surface. The dashed and solid curves indicate the phase- $T$ relation of Models I and II, respectively. 
that the phase difference between $E_{y}$ and $H_{x}$ is always $\pi / 4$.

It is interesting to note that the phase difference shown in Fig. 3 is quite close to $\pi / 4$ for both Models I and II except for extremely short periods. For a long-period variation, of which the skin depth is considerably larger than the undulation amplitude, the conductor approximately behaves as a semi-infinite one having a flat surface. In the case of a shorter-period variation, for which the skin depth is comparable to the undulation amplitude, however, the surface undulation plays an important role in the electromagnetic induction process, so that the phase difference largely deviates from $\pi / 4$ as can be seen for the shortperiod portions shown in Fig. 3.

Such a surface effect is also seen in Fig. 4 in which the transfer function

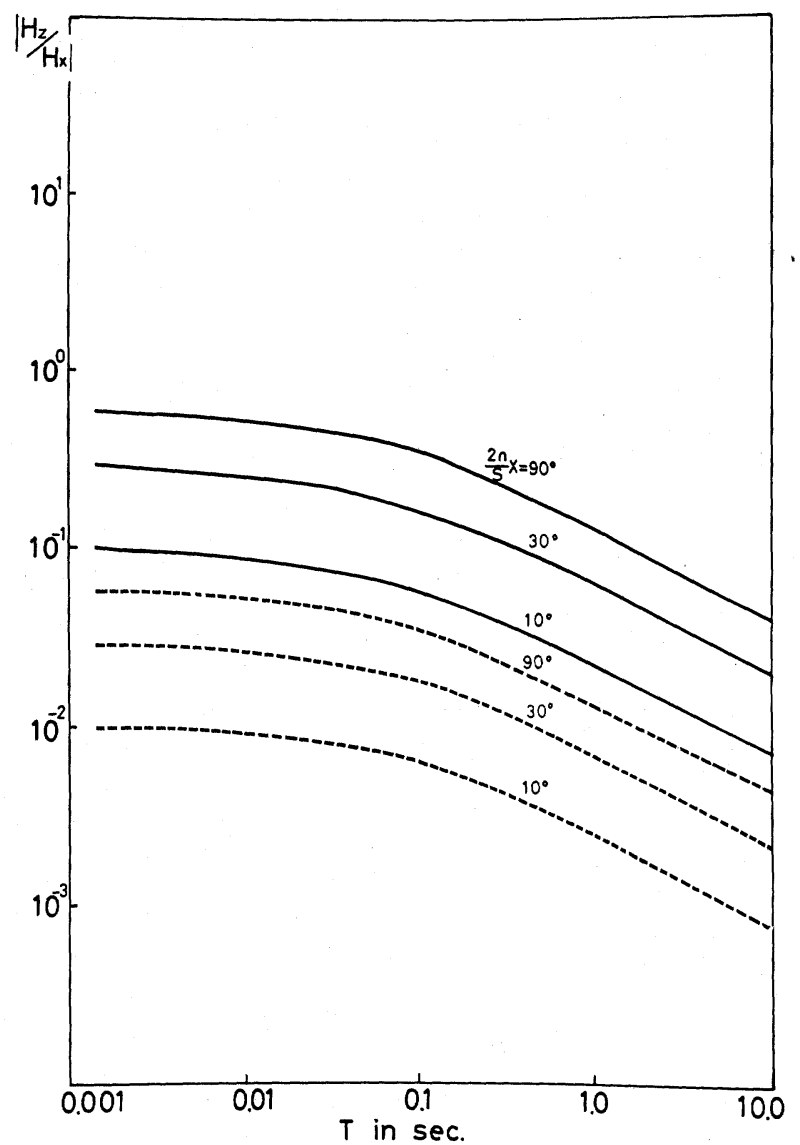

Fig. 4. The absolute value of transfer function vs. $T$ curves at various points on the earth's surface. The dashed and solid curves indicate the transfer function vs. $T$ relation for Models $I$ and II, respectively. 
defined by Eq. (14) vs. $T$ curves at $(2 \pi / s) x=10^{\circ}, 30^{\circ}$ and $90^{\circ}$ of the two models are shown. The $z$-component of the magnetic field, $H_{z}$, is entirely an induced one. On the other hand, $x$-component, $H_{x}$ mainly consists of the inducing field. Therefore, it is natural that $\left|H_{z} / H_{x}\right|$ takes on a smaller value as $T$ becomes longer.

\section{Discussion and Conclusions}

Electromagnetic induction by a uniform inducing field in a semi-infinite earth having an undulating surface is studied using Fourier series expression for scalar and vector potentials of magnetic field.

As a result, it is concluded that the apparent resistivity is affected only slightly by the undulating surface of which the wavelength and peak-to-peak amplitude are 10.0 and $2.0 \mathrm{~km}$, respectively, as long as a resistivity amounting to $100 \mathrm{ohm}$ meter is assumed for the earth. It is noticeable, however, that there is a slight tendency that $\rho_{\mathrm{a}}$ for a $T$ in a range from 0.04 to $10.0 \mathrm{~s}$ takes on a relatively lower value at a point on the depressed portion of the undulating earth's surface (see Fig. 2). On the other hand, the transfer function is considerably affected by the undulation of the earth's surface.

In short, the conclusions obtained from the present study on electromagnetic induction in a semi-infinite medium of finite conductivity having an undulatory surface are summarized as follows;

1) A conventional MT method conducted over the undulating surface of a semi-infinite earth seems to provide an approximately correct resistivity provided typical values such as adopted in this paper are assumed for the resistivity, undulation wavelength and amplitude. It may be said that MT technique is insensitive to a surface undulation of the earth. The $\rho_{\mathrm{a}}-T$ relations are more or less the same from observation point to observation point although $\rho_{\mathrm{a}}$ for longer periods takes on a relatively lower value over the depressed portion of the earth's surface. The phase difference between electric and magnetic fields at any portion of the surface is almost equal to $\pi / 4$ except for extremely short periods.

2) The transfer function vs. $T$ relations differ from place to place depending upon the position of observation point relative to the surface topography.

In spite of the above conclusion, we still have some problems in interpreting the results of conventional MT measurement carried out over the surface of the earth having a highly complex underground structure. Since all layers in the earth are mutually coupling in a problem of electromagnetic induction, an anomalous shape of a deep layer such as suggested in the upper mantle beneath Japan (RIKITAKE, 1969, 1975) may, to some extent, affect the MT interpretation. Such a point should further be studied although highly complicated mathematics will necessarily be involved in the study.

Computations involved in this paper were made on a HITAC M280H system at the Computer Center, University of Tokyo. 


\section{REFERENCES}

Research Group for Crustal Resistivity Structure, Japan, Preliminary report on a study of resistivity structure beneath the Northern Honsyu of Japan, J. Geomag. Geoelectr., 35, 589-608, 1983.

RIKITAKE, T., Electromagnetic induction in a semi-infinite conductor having an undulatory surface, Bull. Earthq. Res. Inst., Univ. Tokyo, 43, 161-166, 1965.

RikitAKE, T., Electromagnetism and the Earth's Interior, Elsevier, Amsterdam, 308 pp., 1966.

RIKITAKE, T., Electromagnetic induction in uniform and non-uniform sheets underlain by an undulating perfect conductor, Bull. Earthq. Res. Inst., Univ. Tokyo, 46, 361-384, 1968.

RiKITAKE, T., The undulation of an electrically conductive layer beneath the islands of Japan, Tectonophysics, 7, 257-264, 1969.

RikitAkE, T., A model of geoelectric structure beneath Japan, J. Geomag. Geoelectr., 27, 233-244, 1975.

RIKITAKE, T. and N. OHSHIMAN, Electromagnetic induction in an irregular layer overlying the earth. The 1st paper: A thin layer over a non-conductor underlain by an undulating perfect conductor, Proc. of the Institute of Natural Sciences, Nihon Univ., 20, 1985 (in press).

Yukutake, T., J. H. Filloux, J. Segawa, Y. Hamano, and H. Utada, Preliminary report on a magnetotelluric array study in the Northwest Pacific, J. Geomag. Geoelectr., 35, 575-587, 1983. 